\title{
Energy Propagation in Linear Hyperbolic Systems.
}

\author{
F. MAINARDI
}

Dipartimento di Fisica dell'Università - Bologna, Italia

INFN - Sezione di Bologna, Italia

\section{E. VAN Groesen}

Department of Applied Mathematics, University of Twente - Twente, The Netherlands

(ricevuto il 24 Agosto 1989)

\begin{abstract}
Summary. - The concept of energy velocity for linear dispersive waves is usually given for a normal mode solution of the system as the ratio between the mean energy flux and the mean energy density. In the absence of dissipation this velocity is known to coincide with the corresponding group velocity. When dispersion is accompanied by dissipation, this interpretation is not correct since the group velocity loses its original meaning and can assume nonphysical values. In this note the relation between energy velocity and group velocity is derived for dissipative, uniaxial waves, governed by a linear hyperbolic system. An example is provided where the energy velocity is compared with the phase and group velocities.
\end{abstract}

PACS 03.40 - Classical mechanics of continuous media: general mathematical aspects.

The concept of energy velocity for linear dispersive waves can be stated as the ratio between the mean energy flux and the mean energy density of a monochromatic wave (normal mode solution of the system), following a classical argument originally due to Reynolds $\left(^{1}\right)$ and Rayleigh $\left(^{2}\right)$ for surface water waves. In the absence of dissipation this velocity is known to coincide with the group

(1) O. REYNOLDS: Nature, 16, 343 (1877).

(2) J. W. Strutt (Lord Rayleigh): Proc. London Math Soc., 9, 21 (1877). 
velocity computed at the frequency (or wavelength) of the wave; this general property of linear systems can be proven in different wayes (see e.g. $\left(^{3-8}\right)$ ).

When there is dissipation this coincidence is manifestly broken since the group velocity (defined by taking the real part in the usual formula) loses its original meaning and can assume, in certain ranges of frequency or wavelength, nonphysical values (e.g., exceeding the wave-front velocity), as pointed out by Sommerfeld and Brillouin $\left({ }^{9}\right)$ for electromagnetic waves. In other words, at variance with conservative systems, for dissipative systems the concept of group velocity may become meaningless and appears not easily related to energy propagation, so that specific treatments are necessarily required.

Recently Mainardi $\left({ }^{10}\right)$ has provided an interesting relation between the energy velocity and the phase velocity for dispersive and dissipative waves that are governed by a linear hyperbolic system. In this note, in view of the importance of the concept of group velocity for conservative waves (in both linear and nonlinear system, see e.g. $\left({ }^{(1)}\right)$ ), we will show how, in the presence of dissipation, the energy velocity of hyperbolic waves can be related to the group velocity as well. Furthermore an example of physical interest is provided where the energy velocity is compared with the phase and group velocity.

As in ${ }^{(1)}$ ) we restrict ourselves to uniaxial waves and we agree to write the governing hyperbolic system in the form

$$
\frac{\partial \boldsymbol{u}}{\partial t}+D \frac{\partial \boldsymbol{u}}{\partial x}+M \boldsymbol{u}=0
$$

where $\boldsymbol{u}=\boldsymbol{u}(x, t)$ is a real $n$-vector function which represents the field variable and $D$ and $M$ are constant real $n \times n$ matrices, with $D$ symmetric. Furthermore we assume that the variable $\boldsymbol{u}$ in eq. (1) is chosen in such a way that the functional

$$
\mathcal{E}[\boldsymbol{u}]=\frac{\boldsymbol{u} \cdot \boldsymbol{u}}{2}
$$

is the energy density for the physical system, where $\cdot$ denotes the inner product in $\boldsymbol{R}^{n}$.

(3) C. G. Rossby: J. Meteorol., 2, 187 (1945).

$\left(^{4}\right)$ L. J. F. BROER: Appl. Sci. Res. A, 2, 329 (1951).

(5) M. A. Bıот: Phys. Rev., 105, 1129 (1957).

(6) G. B. Whitham: Commun. Pure Appl. Math., 14, 675 (1961).

(?) G. B. Whitham: J. Fluid Mech., 22, 273 (1965).

(8) M. J. Sir LightHill: J. Inst. Math. Applic., 1, 1 (1965).

$\left(^{9}\right)$ L. BRILlouin: Wave Propagation and Group Velocity (New York, N.Y., 1960), Chapt. 2, 5.

${ }^{\left({ }^{10}\right)}$ F. MAINARdi: Wave Motion, 9, 201 (1987).

(') G. B. Whitham: Linear and Nonlinear Waves (New York, N.Y., 1974), Chapt. 5, 15. 
The system is supposed to be nonconservative in the sense that $\&$ satisfies the following balance law:

$$
\frac{\partial \mathscr{O}}{\partial t}+\frac{\partial \mathscr{T}}{\partial x}=-\mathcal{f}
$$

where $\mathscr{T}=\mathscr{F}[\boldsymbol{u}]$ is the (energy) flux density and $\mathcal{S}=\mathscr{S}[\boldsymbol{u}]$ is the rate of loss (if positive), or of supply (if negative). We agree to refer to $\mathcal{f}$ as to the loss density. For a detailed discussion of the energy balance law in nonconservative systems we refer to recent papers of the authors $\left({ }^{12,13}\right)$, where also the notion of centrovelocity of energy is introduced.

For the system (1) we obtain particularly simple expressions for the flux and for the loss $\left(^{10,12}\right)$; in the present notation they read

$$
\begin{aligned}
& \mathscr{F}[\boldsymbol{u}]=\frac{\boldsymbol{u} \cdot D \boldsymbol{u}}{2}, \\
& \mathcal{S}[\boldsymbol{u}]=\boldsymbol{u} \cdot M^{\S} \boldsymbol{u},
\end{aligned}
$$

where $M^{\mathrm{s}}\left(M^{\mathrm{a}}\right)$ denotes the symmetric (antisymmetric) part of the constant matrix $M$.

Let us now recall the relevant concepts related to energy propagation. Denoting by $\langle f[\boldsymbol{u}]\rangle$ the space mean value of a density $f=f(x, t)$ for a prescribed solution $\boldsymbol{u}$, computed in a fixed interval of length $L$ (say, in $x_{0} \leqslant x \leqslant x_{0}+L$ ), and assuming $\mathscr{F}\left(x_{0}, t\right)=\mathscr{F}\left(x_{0}+L, t\right) \forall t$, we introduce the following (time dependent) functionals, the energy-flux velocity:

$$
V_{\mathrm{e}}[\boldsymbol{u}]=\frac{\langle\mathscr{F}[\boldsymbol{u}]\rangle}{\langle\mathscr{E}[\boldsymbol{u}]\rangle}
$$

and the dissipation rate

$$
\alpha[\boldsymbol{u}]=\frac{\langle\cdot \rho[\boldsymbol{u}]\rangle}{\langle\varrho[\boldsymbol{u}]\rangle} .
$$

The velocity $V_{\mathrm{e}}$ is sometimes called the mean velocity of energy transport; here, we will simply refer to it as the energy velocity. The importance of the rate $\alpha$ can be recognized after integrating (3) over $x$. It follows that

$$
\frac{\mathrm{d}}{\mathrm{d} t}\langle g\rangle=-\alpha\langle g\rangle
$$

(12) E. Van Groesen and F. Mainardi: Wave Motion, 11, 201 (1989).

$\left.{ }^{13}\right)$ E. VAN Groesen and F. Mainardi: Balance Laws and Centrovelocity in Dissipative Systems, to be published. 
from which it is found that

$$
\langle\mathscr{G}[\boldsymbol{u}]\rangle=\left\langle\mathscr{E}_{0}\right\rangle \exp \left[-\int_{0}^{t} \alpha[\boldsymbol{u}(\tau)] \mathrm{d} r\right]
$$

where $\mathcal{G}_{0}$ is the energy density corresponding to the initial data $\boldsymbol{u}(x, 0)$.

As a consequence of eqs. (2) and (4)-(7) we get

$$
\begin{aligned}
& V_{\mathrm{e}}[\boldsymbol{u}]=\frac{\langle\boldsymbol{u} \cdot D \boldsymbol{u}\rangle}{\langle\boldsymbol{u} \cdot \boldsymbol{u}\rangle}, \\
& \alpha[\boldsymbol{u}]=2 \frac{\left\langle\boldsymbol{u} \cdot M^{\mathrm{s}} \boldsymbol{u}\right\rangle}{\langle\boldsymbol{u} \cdot \boldsymbol{u}\rangle} .
\end{aligned}
$$

From eqs. (2), (5) we note that, if the matrix $M^{\mathrm{s}}$ is proportional to the identity matrix, then, for any solution $\boldsymbol{u}(x, t)$ of (1) and for all $x, t$, the loss density is proportional to the energy density. This peculiar fact, referred to as uniform damping, can also be expressed by

$$
\alpha=\frac{\mathscr{S}}{\mathscr{G}}=\frac{\langle\mathscr{S}\rangle}{\langle\mathscr{G}\rangle},
$$

where now the dissipation rate $\alpha$ is a characteristic constant of the system, indipendent of the solution and of the space domain $\left({ }^{12}\right)$.

In this paper we consider particular space-periodic solutions, referred to as normal modes, from which usually the analysis of linear dispersive waves starts. These solutions are of the form

$$
\boldsymbol{u}(x, t ; k)=\operatorname{Re}[\boldsymbol{V}(k) \exp [i[k x-\omega(k) t]]]
$$

with $k$ real and $\omega$ complex in general, and $\boldsymbol{V}$ a complex $n$-vector. Henceforth we will denote a given normal mode simply by $\boldsymbol{u}(k)$, so dropping the dependence on $x, t$; furthermore we will use the notation $\boldsymbol{u}^{\mathrm{c}}(k)$ to denote the complex solution whose real part provides $\boldsymbol{u}(k)$.

These normal modes represent (pseudo) monochromatic waves, since they are sinusoidal in space with period (wavelength) $\lambda=2 \pi / k$, but not necessarily sinusoidal in time, since $\omega$ may be complex. Only if $\omega$ is real, these solutions represent effectively monochromatic waves with a time period $T=2 \pi / \omega$ and with a constant amplitude. In general these waves are decaying or growing exponentially in time if the quantity (damping factor)

$$
\gamma=-\operatorname{Im}[\omega]
$$


is positive or negative, and they propagate with speed (phase velocity)

$$
V_{\mathrm{p}}=\frac{\operatorname{Re}[\omega]}{k} .
$$

For $\boldsymbol{u}(k)$, and hence $\boldsymbol{u}^{\boldsymbol{c}}(k)$, to be a solution of (1), $\omega$ and $\boldsymbol{V}$ depend on $k$ in such a way that they satisfy the following eigenvalue problem:

$$
[-i \omega I+i k D+M] \boldsymbol{V}=0,
$$

namely they should be an eigenvalue and a corresponding eigenvector of the matrix $\Omega(k)=k D-i M$. In particular $\omega$ must satisfy the dispersion relation that follows from (16), i.e.

$$
\mathscr{D}(\omega, k) \equiv \operatorname{det}[-i \omega I+i k D+M]=0 .
$$

In general at a given $k$ there may be several branches $\omega(k)$ of (17), providing distinct modes. For a given branch $\omega=\omega(k)$, the dispersive properties are illustrated by the functions (14) and (15). Furthermore we introduce the group velocity as

$$
V_{\mathrm{g}}=\frac{\mathrm{d}}{\mathrm{d} k} \operatorname{Re}[\omega(k)] .
$$

In the conservative case $\omega$ is real and $V_{\mathrm{g}}$ has the kinematic meaning of speed of the group (wave packet). In the nonconservative case, $\operatorname{Im}[\omega(k)]$ does not vanish in general so that $V_{\mathrm{g}}$ is expected to lose its usual meaning (see $e . g .\left(^{9}\right)$ ), while $V_{\mathrm{p}}$ does keep it.

The energy velocity and the attenuation factor associated with the waves (13) can easily be obtained from eqs. (10), (11), respectively, inserting the solution (13) and averaging over the wavelength. For this purpose we recall the following properties concerning two normal modes with a given $k$ and $\omega(k)$ but with different amplitude:

$$
2\left\langle\boldsymbol{u}_{1}(k) \cdot \boldsymbol{u}_{2}(k)\right\rangle=\operatorname{Re}\left[\left\langle\boldsymbol{u}_{1}^{\mathrm{c}}(k) \cdot \boldsymbol{u}_{2}^{\mathrm{c}}(k)\right\rangle\right]
$$

and

$$
\left\langle\boldsymbol{u}_{1}^{\mathrm{c}}(k) \cdot \boldsymbol{u}_{2}^{\mathrm{c}}(k)\right\rangle=\boldsymbol{u}_{1}^{\mathrm{c}}(k) \cdot \boldsymbol{u}_{2}^{\mathrm{c}}(k)=\exp [-2 \gamma t]\left(\boldsymbol{V}_{1} \cdot \boldsymbol{V}_{2}\right),
$$

where the inner product is in $\boldsymbol{R}^{n}$ or $\boldsymbol{C}^{n}$ as appropriate.

The importance of relations (19)-(20) is that they enable us to compute $V_{\mathrm{e}}$ and $\alpha$ without carrying out the averages present in eqs. (10)-(11); we obtain

$$
V_{\mathrm{e}}=\frac{\boldsymbol{V} \cdot D \boldsymbol{V}}{\boldsymbol{V} \cdot \boldsymbol{V}}
$$


and

$$
\alpha=2 \frac{\boldsymbol{V} \cdot M^{\mathrm{s}} \boldsymbol{V}}{\boldsymbol{V} \cdot \boldsymbol{V}}
$$

From (21)-(22) we recover the property that for normal-mode solutions the energy velocity and the dissipation rate are constant (time independent), depending uniquely on the wavenumber. Furthermore, the energy velocity turns out to be bounded from above and below by the extreme eigenvalues of the matrix $D$, i.e. by the extreme characteristic velocities of the hyperbolic system.

Recently Mainardi $\left({ }^{10}\right)$ has proven the following identities:

$$
V_{\mathrm{e}}=V_{\mathrm{p}}+\frac{i}{k} \frac{\boldsymbol{V} \cdot \boldsymbol{M}^{\mathrm{a}} \boldsymbol{V}}{\boldsymbol{V} \cdot \boldsymbol{V}}
$$

and

$$
\gamma=\frac{\boldsymbol{V} \cdot M^{\mathrm{s}} \boldsymbol{V}}{\boldsymbol{V} \cdot \boldsymbol{V}}
$$

These equations can readily be obtained taking the $\boldsymbol{C}^{n}$-inner product of (16) with $\boldsymbol{V}$, and considering the imaginary and real parts, respectively. From (22) and (24), we may incidentally note that $\alpha=2 \gamma$.

Previously, Broer and Peletier $\left({ }^{14}\right)$ have used the eigenvalue equation (16) to prove that, in the absence of dissipation $\left(M^{\mathrm{s}}=0\right)$, the r.h.s. of (21) equals the group velocity. Now we would like to extend the analysis in $\left({ }^{10,14}\right)$ to show how the energy velocity can be related to the group velocity, taking into account the symmetric part $M^{\mathrm{s}}$ of the matrix $M$, that is responsible for dissipation. This relation, which is known to be an identity when $M$ is purely antisymmetric $\left({ }^{14}\right)$, can be obtained from the eigenvalue equation (16) after some manipulations. For this purpose we differentiate (16) with respect to $k$ and take the $\boldsymbol{C}^{n}$ inner product with $\boldsymbol{V}$ so obtaining

$$
\omega^{\prime} \boldsymbol{V} \cdot \boldsymbol{V}-\boldsymbol{V} \cdot D \boldsymbol{V}+\boldsymbol{V} \cdot(\omega I-k D+i M) \boldsymbol{V}^{\prime}=0, \quad \omega^{\prime}=\frac{\mathrm{d} \omega}{\mathrm{d} k}, \quad \boldsymbol{V}^{\prime}=\frac{\mathrm{d} \boldsymbol{V}}{\mathrm{d} k},
$$

and hence

$$
\omega^{* \prime} \boldsymbol{V} \cdot \boldsymbol{V}-\boldsymbol{V} \cdot D \boldsymbol{V}+\boldsymbol{V}^{\prime} \cdot\left(\omega^{*} I-k D-i M^{\dagger}\right) \boldsymbol{V}=0
$$

where $*$ and ${ }^{\dagger}$ denote complex and Hermitian conjugate, respectively. Noting that

$$
\omega^{*}=\omega+2 i \gamma, \quad M^{\dagger}=-M+2 M^{\mathrm{s}},
$$

(14) L. J. F. Broer and L. A. Peletier: Appl. Sci. Res. A, 17, 65 (1967). 
and, using (16), eq. (26) reads

$$
\omega^{* \prime} \boldsymbol{V} \cdot \boldsymbol{V}-\boldsymbol{V} \cdot D \boldsymbol{V}-2 i \boldsymbol{V}^{\prime} \cdot\left(M^{\mathrm{s}}-\gamma I\right) \boldsymbol{V}=0
$$

namely

$$
\frac{\boldsymbol{V} \cdot D \boldsymbol{V}}{\boldsymbol{V} \cdot \boldsymbol{V}}=\omega^{* \prime}-\frac{2 i}{\boldsymbol{V} \cdot \boldsymbol{V}}\left[\boldsymbol{V} \cdot\left(M^{\mathrm{s}}-\gamma I\right) \boldsymbol{V}^{\prime}\right]^{*}
$$

Using (21) and noting that $\omega^{\prime}=V_{\mathrm{g}}-i \gamma^{\prime}$, it is a simple matter to get from (29)

$$
\boldsymbol{V}_{\mathrm{e}}=V_{\mathrm{g}}-\frac{2}{\boldsymbol{V} \cdot \boldsymbol{V}} \operatorname{Im}\left[\boldsymbol{V} \cdot\left(M^{\mathrm{s}}-\gamma I\right) \boldsymbol{V}^{\prime}\right]
$$

and

$$
\frac{\mathrm{d} \gamma}{\mathrm{d} k}=\frac{2}{\boldsymbol{V} \cdot \boldsymbol{V}} \operatorname{Re}\left[\boldsymbol{V} \cdot\left(M^{\mathrm{s}}-\gamma I\right) \boldsymbol{V}^{\prime}\right]
$$

We recognize from (30) the fact that the identification of energy velocity with group velocity is valid not only in the absence of dissipation $\left(\gamma=0 \Leftrightarrow M^{\mathrm{s}}=0\right)$, but also for uniform damping $\left(M^{s}=\gamma I\right)$. In the particular case of uniform damping without dispersion $\left(M=M^{s}=\gamma I\right)$ we recover from (23), (30) the trivial equality of the three velocities $V_{\mathrm{e}}, V_{\mathrm{p}}, V_{\mathrm{g}}$.

We conclude our analysis with recalling the instructive example of the linear Klein-Gordon equation with dissipation (KGD equation) that rules wave propagation in an elastic string, anchored elastically to its equilibrium position by a transverse restoring force and damped by air friction. It is a second-order hyperbolic equation that reads

$$
\dot{\phi}_{t t}+2 a \dot{\phi}_{t}+b^{2} \phi=c^{2} \phi_{x x}
$$

where $\phi=\phi(x, t)$ denotes the transverse displacement and $a, b, c$ are nonnegative constants related with air damping, restoring force and string tension, respectively $\left({ }^{15}\right)$. The corresponding dispersion relation reads

$$
(\omega+i a)^{2}=c^{2} k^{2}+\left(b^{2}-a^{2}\right),
$$

and indicates that different cases can be expected, depending on the sign of $\left(b^{2}-a^{2}\right)$. If $0 \leqslant a \leqslant b$, then for any $k$ the real part of $\omega$ does not vanish, i.e. there is propagation for each $k$; if $0 \leqslant b<a$, there is propagation only for wavenumbers

( $\left.{ }^{15}\right)$ G. R. BALDOCK and T. BRIDGEMAN: The Mathematical Theory of Wave Motion (Chichester, U.K., New York, N.Y., 1981), Chapt. 5. 
$k$ such that $|k| \geqslant \sqrt{a^{2}-b^{2}}$. The propagation regimes are characterized by a constant damping factor $\gamma=a$ and

$$
V_{\mathrm{p}}=\frac{\sqrt{c^{2} k^{2}+\left(b^{2}-a^{2}\right)}}{k}, \quad V_{\mathrm{g}}=\frac{c^{2}}{V_{\mathrm{p}}} .
$$

In particular we get normal dispersion $\left(0 \leqslant V_{\mathrm{g}} \leqslant c \leqslant V_{\mathrm{p}}\right)$ if $0 \leqslant a<b$, anomalous dispersion $\left(0 \leqslant V_{\mathrm{p}} \leqslant c \leqslant V_{\mathrm{g}}\right)$ if $0 \leqslant b<a$, and no dispersion $\left(V_{\mathrm{p}}=V_{\mathrm{g}}=c\right)$ if $a=b$.

The energy velocity has been computed in $\left.{ }^{(10,15}\right)$ starting from the energy balance for a single-mode solution. The terms $\mathscr{G}, \mathscr{F}$ and $\mathcal{S}$ entering this balance are, respectively,

$$
\mathscr{E}=\left(\phi_{t}^{2}+c^{2} \phi_{x}^{2}+b^{2} \phi^{2}\right) / 2, \quad \mathscr{F}=-c^{2} \phi_{x} \phi_{t}, \quad \mathcal{S}=2 a \phi_{t}^{2},
$$

and the energy velocity turns out to be

$$
V_{\mathrm{e}}=\operatorname{Re}[\omega] \frac{c^{2} k}{b^{2}+c^{2} k^{2}},
$$

or, using (33)-(34),

$$
V_{\mathrm{e}}=V_{\mathrm{p}}\left[1-\frac{b^{2}}{b^{2}+c^{2} k^{2}}\right]=V_{\mathrm{g}}\left[1-\frac{a^{2}}{b^{2}+c^{2} k^{2}}\right] .
$$

Formulae (36) and (37) can also be found after some elementary calculations from eq. (21) or (23) or (30) as well, thus providing a check of our present theory. For convenience we quote the relevant passage required to get the energy velocity.

Choosing $\boldsymbol{u}=\operatorname{col}\left(\phi_{t}, c \phi_{x}, b \phi\right)$, the KGD equation (32) can be written as a firstorder hyperbolic system of type (1)-(2) with $n=3$, where

$$
D=\left[\begin{array}{ccc}
0 & -c & 0 \\
-c & 0 & 0 \\
0 & 0 & 0
\end{array}\right), \quad M=\left[\begin{array}{ccc}
2 a & 0 & b \\
0 & 0 & 0 \\
-b & 0 & 0
\end{array}\right)
$$

After deriving the dispersion relation (33) from the eigenvalue problem (16) using (1), (13), (38) (and neglecting the spurious eigenvalue $\omega=0$ that arises because a three-vector $\boldsymbol{u}$ is introduced to describe a second-order equation), we obtain the eigenvector for the progressive mode, to be inserted in eqs. (21), (23), (30),

$$
\boldsymbol{V}=\operatorname{col}(\omega,-c k, i b) \text {. }
$$



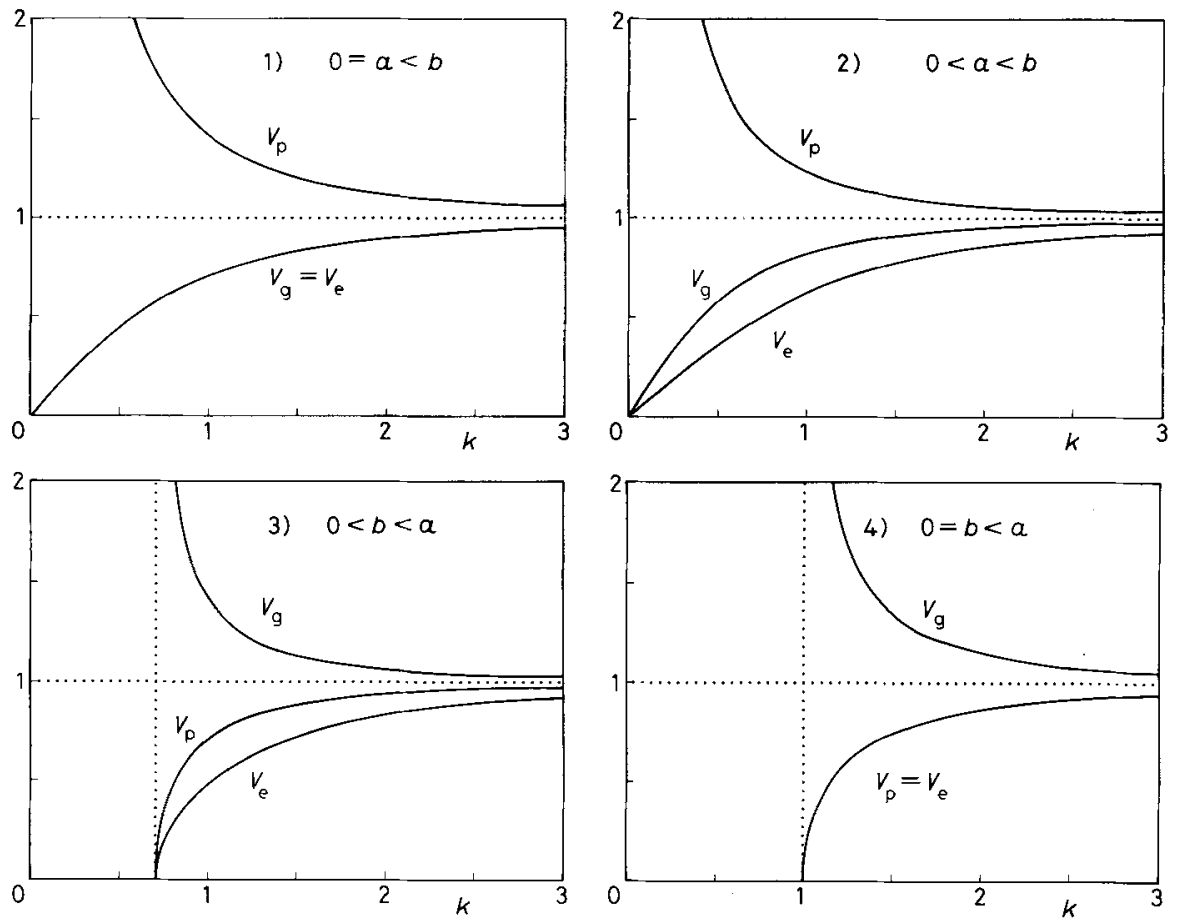

Fig. 1. - Comparison among the energy velocity $V_{\mathrm{e}}$, the phase velocity $V_{\mathrm{p}}$ and the group velocity $V_{\mathrm{g}}$ for the linear KGD equation $\phi_{t t}+2 a \phi_{t}+b^{2} \phi=c^{2} \phi_{x x}$, with $c=1$ and 1) $a=0$, $b=1$; $) a=1 / \sqrt{2}, b=1$; 3) $a=1, b=1 / \sqrt{2}$; 4) $a=1, b=0$.

In particular, after simple algebra, we obtain the relevant expressions

$$
\left\{\begin{array}{l}
\boldsymbol{V} \cdot \boldsymbol{V}=\omega \omega^{*}+b^{2}+c^{2} k^{2}=2\left(b^{2}+c^{2} k^{2}\right), \\
\boldsymbol{V} \cdot D \boldsymbol{V}=c^{2} k\left(\omega+\omega^{*}\right)=2 \operatorname{Re}[\omega] c^{2} k=2 c^{2} k^{2} V_{\mathrm{p}}, \\
\boldsymbol{V} \cdot M^{\mathrm{a}} \boldsymbol{V}=i b^{2}\left(\omega+\omega^{*}\right)=2 i b^{2} \operatorname{Re}[\omega]=2 i b^{2} k V_{\mathrm{p}}, \\
\boldsymbol{V} \cdot\left(M^{\mathrm{s}}-\gamma I\right) \boldsymbol{V}^{\prime}=a\left(\omega^{*} \omega^{\prime}-c^{2} k\right)=i a^{2} V_{\mathrm{g}} .
\end{array}\right.
$$

We note that $V_{\mathrm{e}}$ as given by (36) and (37) is an increasing function of $k$ which does not exceed the wave front velocity $c$ (either $V_{\mathrm{p}}$ or $V_{\mathrm{g}}$ ), and that it reduces to the phase velocity only if $b=0$, and to the group velocity only if $a=0$. In the particular case $a=b$, it remains distinct from $c$ in spite of the absence of dispersion (dissipation is however acting not uniformly on the energy since $M^{s}$ is not proportional to $I$ ).

For the sake of clearness, in fig. 1 we show pictures that compare the energy velocity $V_{\mathrm{e}}$ with the kinematic velocities $V_{\mathrm{p}}, V_{\mathrm{g}}$ in the following relevant cases: 1) $0=a<b$, 2) $0<a<b, 3) 0<b<a$,4) $0=b<a$. 
This work was supported in part by C.N.R., Gruppo Nazionale per la Fisica Matematica, by I.N.F.N., Sezione di Bologna, and by Italian Ministry of Education (MPI: 60\% grants). EVG wishes to thank C.N.R. for providing the opportunity to visit the University of Bologna. We also thank G. Vitali for his assistance with computing. 